\title{
EFFECTS OF X-RAY ON FIBROBLAST MECHANICAL PROPERTIES
}

\author{
Ashkan Heydarian, Siamak Khorramymehr \\ Department of Biomedical Engineering, Science and Research Branch, Islamic Azad University, Tehran, Iran \\ e-mail: s.khorramymehr@srbiau.ac.ir \\ Behnoosh Vasaghi-Gharamaleki \\ Department of Basic Sciences of Rehabilitation, Iran University of Medical Sciences (IUMS), Iran
}

\begin{abstract}
Concerning the widespread use of X-rays to detect various diseases, such as oral and dental ones, it is essential to study the effects of this radiation on living cells. From the past, genetic effects and cell death because of X-rays have been studied. In addition, the effect of this ionizing radiation on the mechanical properties of the cell and cytoskeleton has been studied, but different results have been obtained based on different models. In this study, post-culture gingival fibroblast cells were classified into two groups of control and radiation with Nano Magnetic Particles functionalized by folic acid. The cells of the radiation group were exposed to X-rays of $3 \mathrm{mGy} \cdot \mathrm{cm}^{2}$. The specimens were undergone static creep test by a magnetic tweezer. Spring and damper coefficients were obtained based on the viscoelastic solid modeling. The static and dynamic stiffness of the groups was also calculated. The maximum deformation was decreased after radiation from $0.049 \pm 0.01 \mu \mathrm{m}$ to $0.029 \pm 0.01 \mu \mathrm{m}$ and the static stiffness of the model was 1.6 times decreased. Also, the gel point frequency for the control group was $27 \mathrm{~Hz}$ and for the radiation group was $7.5 \mathrm{~Hz}$. The results show that the static and dynamic stiffness of the cells decreases after radiation, and less deformation appears in the cells after irradiation. These changes can be due to the breakdown of membrane chemical bonds and activation of actin fibers after radiation.
\end{abstract}

Keywords: viscoelastic, biomechanics, cellular mechanics, X-ray effects

\section{Introduction}

Radiation of ionizing rays, especially radiations emitted from radioactive materials, plays a vital role in medicine. Nowadays, the use of these radiations at various stages, including the diagnosis and treatment of diseases, has increasingly contributed to the advancement of medical science. Ionizing radiation is one of the most important and practical forms that has many applications in the treatment and diagnosis of various diseases. Among the most commonly used technologies, there are radiology for imaging damaged hard tissue, CT scan for imaging soft tissues and cancer tumors, fluoroscopy for imaging living tissues and radiotherapy for cancer treatment (Health and Physics, 2009). Despite the benefits of X-rays to detect and treat diseases, the X-ray radiation has considerable potential for penetration into living tissues due to its high energy, which can have an adverse effect on living cells or tissues, or lead to an abnormal function. According to the International Commission of Radiological Protection (ICRP, 2007), the effects of these radiations are divided into two types: definitive effects (if the radiation dose is much higher than the allowable limit, which can lead to reaction in tissues of the body) and possible effects (which are more known as the effects on DNA of cells and can lead to harmful effects, including cancer) (Valentin, 2007). These negative effects depend on different factors such as the type of radiation, radiation rate, type of tissue or the cell receiving radiation and the dosage of radiation (Verma et al., 2016). Many previous papers and studies suggest negative effects of X-rays (Risi et al., 2012). 
One of the radiations applied to the body through the use of medical devices is the X-ray for Panoramic Radiology. This technique is widely used today for preparation of flat images from curved surfaces of tooth and jaw. This method is an effective way to check teeth arrangement and their health status (Boeddinghaus and Whyte, 2008). Recently, it has been found in some articles and scientific studies that X-rays radiated from this device can have an adverse effect on tissues and cells of the human body. For example, Preethi et al. (2016) found in their study on the impact of Panoramic Radiology and its effect on Genotoxic that this X-ray imaging method can increase formation of micronuclei in the cell membrane. In fact, micronuclei and their increasing production in the cell are indicative of the increased cell death (Preethi et al., 2016). Also, EMM Cerqueira et al. (2008) addressed in another study the effect of X-ray radiated on epithelial cells of gum during Panoramic Radiology. Their results also indicate the impact of this radiation on the increase in cell death (Cerqueira et al., 2008). In addition to mentioned studies and the adverse effects of X-rays on DNA and the cell structure, a number of current studies and papers show the effects of X-rays on mechanical properties of cells and tissues in the body. Thomas et al. (2003) studied the effect of X-rays with three different doses of 0 (control), 25 and 50 grays on blood cells (platelets and lymphocytes) and found that the increased radiative dose of X-rays can lead to increased cellular rigidity. They also found that Young's modulus of irradiated platelets increased compared to the control group, and the viscosity of the cytoplasm of the irradiated lymphocytes increased compared to the control group (Thomas et al., 2003). In another study, Panzetta et al. (2017) investigated the effect of X-rays with the intensity of 1 and 2 Gy on SV40 and BALB/c3T3 cells. According to their study, mechanical properties of the skeleton of the cells under radiation have increased (Panzetta et al., 2017). The mechanical properties of the cells are important because, along with chemical properties, they can be considered as a marker for diagnosis of cell health. Due to the importance of this fact, we know today that the advent of various diseases causes changes in chemical and mechanical properties of the cells. Hence, these mechanical changes can be recognized as an indicator for the diagnosis of a damaged cell from the healthful one (Rianna and Radmacher, 2016; Rosenbluth et al., 2008). One of the mechanical properties whose change can be related to structural changes resulting from the damaged or ill cell are the viscoelastic properties of the cell (Bao and Suresh, 2003; Ingber, 2002).

Therefore, we have investigated the effect of X-ray emitted in Periapical Radiology on the mechanical properties (viscoelastic) of gingival fibroblastic cells by Magnetic Tweezer Cytometry (MTC). Our assumption in this paper is that, firstly, because X-ray radiation is an ionizing one, it can make changes in the cell structure and proteins of the cytoskeleton, thereby, decreases the stiffness and increases deformation. In this research, we used Nano Magnetic Particles (NMP) instead of Micro Magnetic $\left(\mathrm{Fe}_{3} \mathrm{O}_{4}\right)$ Particle (MMP) because of some defects which are originated by MMP detection like particles aggregation and glass binding. Using NMP can optimize MTC for cells structural properties gaining. Also previously, MNP were used for cell manipulation and cell behavior remote control (Dobson, 2008; Kalinin et al., 2002) and were utilized for investigation of subcellular mechanical properties (Overby et al., 2005; Kanger et al., 2008; Pan et al., 2012).

\section{Materials and methods}

\subsection{Cell line}

Cellular specimens in this experiment were human gingival fibroblasts (HGF). The cells have been initially cultured at Iranian biological research center (IBRC) in $1.5 \mathrm{ml}$ DMEM (Dulbecco's Modified Eagle's medium), 20\% FCS (Fetal Calf Serum) with $7 \%$ magnetic nanoparticle solution functionalized by folic acid at concentration of $1000 \mathrm{ppm}$ and $10 \mathrm{~nm} \pm 5 \mathrm{~nm}$ in size for 4 hours at a temperature of $26.5^{\circ} \mathrm{C}$ and in the presence of $5 \% \mathrm{Co}_{2}$ in 10 dishes with $35 \mathrm{~mm}$ diameter. After 
the number of cells in each of the Petri dishes reached $3 \cdot 10^{6}$, the cells were removed from the culture and after freezing transferred to the laboratory for the other stages of the experiment. In the next step, out of 10 Petri dishes, 5 were assigned to the normal group and 5 were allocated to the radiation group. At the last stage, the specimen was defreezed by incubator at $37^{\circ} \mathrm{C}$ and transferred to the Magnetic Tweezer system for testing.

\subsection{The source of X-ray}

In this experiment, Periapical Radiology (Helio Dent Plus, Sirona) in Dr. F. Momeni Maxillofacial Radiology Center was used. The device has radiated X-ray dose of $3 \mathrm{mGy} \cdot \mathrm{cm}^{2}$ with a voltage of $60 \mathrm{kV}$ and a period of 0.02 second on specimens. The radiation dose was calculated from the dental periapical radiography.

\subsection{Magnetic Tweezer}

A Magnetic Tweezer was used for the test. The method and mechanical load applied by the device to the cells have been mentioned in references and previous studies (Kollmannsberger and Fabry, 2007). The magnetic tweezer system used in this study includes the following parts: the magnetic tip, coil holder and a coil with a ferrous metal core with a 1500 rounds copper wire with $0.7 \mathrm{~mm}$ diameter. A pulse generator (AFG3000, GW Instek, Taiwan) was also used to create the required mechanical pulse. The device imaging part also includes a microscope (KM1000, CHINA) and a camera (5 Megapixels, 60 frames per second) for recording sample images and videos.

\subsection{Calibration}

In order to ensure the accuracy of the results, the device was calibrated prior to the testing stages according to the methods described in previous studies and references (Tanase et al., 2007).

\subsection{Test procedure}

Initially, the media of normal cells and radiated cells were evacuated by the micropipette. Four cells were collected in each dish randomly. Totally, 20 cells were tested in each group. The samples undergone pre-conditioning before the test. For this purpose, preloading was performed during 10 cycles with $2.5 \mathrm{~s}$ of loading and $2.5 \mathrm{~s}$ of unloading. In the next step, the samples of both groups were loaded and the constant force of $299 \mathrm{pN}$ was applied to the samples for $2.5 \mathrm{~s}$. A cell was selected as a sample from each of the Petri dishes. Then, using the recorded image processing of each sample, the amount of cell membrane displacement was obtained during the testing period. Processing of the recorded images of the samples was performed by open source software (TRACKER4.92). The extracted data were analyzed statistically for each sample and the mean results of each sample were sorted. Then the mean displacement-time graphs were plotted with their standard deviations. Also, the displacement data at the same time for each sample were studied statistically. These data had were distributed on the normal graph for each cell according to the Anderson-Darling test. In order to determine the behavior of the specimens, the creep slope of the mean displacement-time diagram was considered as an indicator for determining the fluid-like or solid behavior of the specimens. After identifying the behavior of the samples, in order to find the closest mechanical model, the data were fitted by Mathematica 9 software, then the model with the least root-mean-square error (RMSE) was selected as the preferred model. In the next step, the unknown parameters of the mechanical model, i.e., the coefficients of spring and damper, were calculated by curve fitting. Then, having known the unknown parameters, the differential equation and the displacement function were obtained. The creep function of each 
cell could be obtained by these equations which represented the static stiffness of the cellular model. In addition, by solving the dynamic response of the differential equations, the loss and storage modules of each group were obtained. Additionally, the numerical value of $D *$ which indicates the dynamic stiffness of the cell was calculated (Fig. 1).

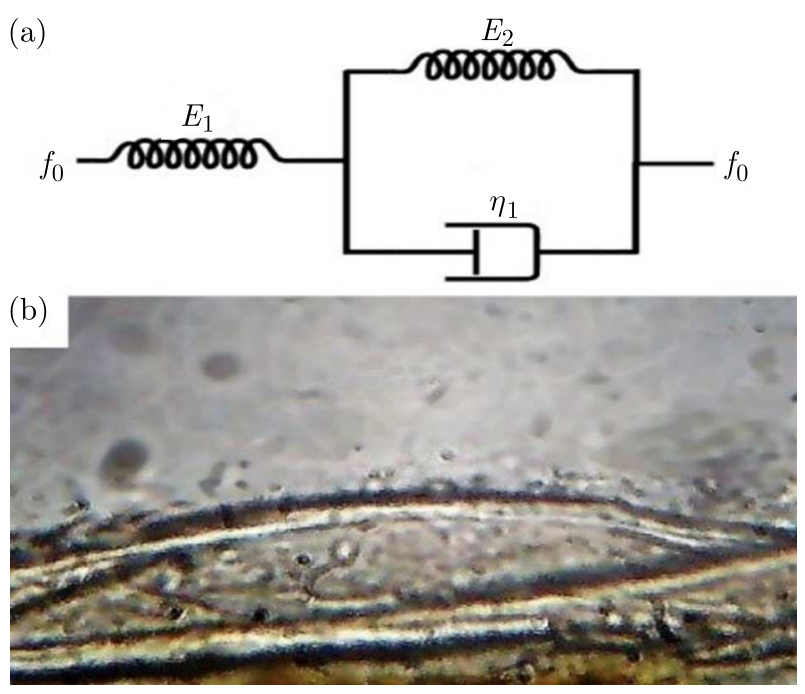

Fig. 1. Schematic illustration of three elements of the Standard Solid Differential model (a) NMPs conjugated with the fibroblast cell (b)

\section{Results}

After extracting the data from each sample video, the mean displacement-time graphs were plotted with standard deviations of each sample (Fig. 2). The graphs indicate the displacement of the cell membrane under mechanical loading. Then, in order to study the behavior of the samples, the creep section of the graphs was determined and then the slope of the creep section was used as a criterion for determining the behavior of the samples. Therefore, the average of both groups exhibits solid behavior. This result is due to zero slope in the creep section of the time-displacement graphs. After determining the behavior of the samples, the best mechanical model and the static and dynamic stiffness were examined.

(a)

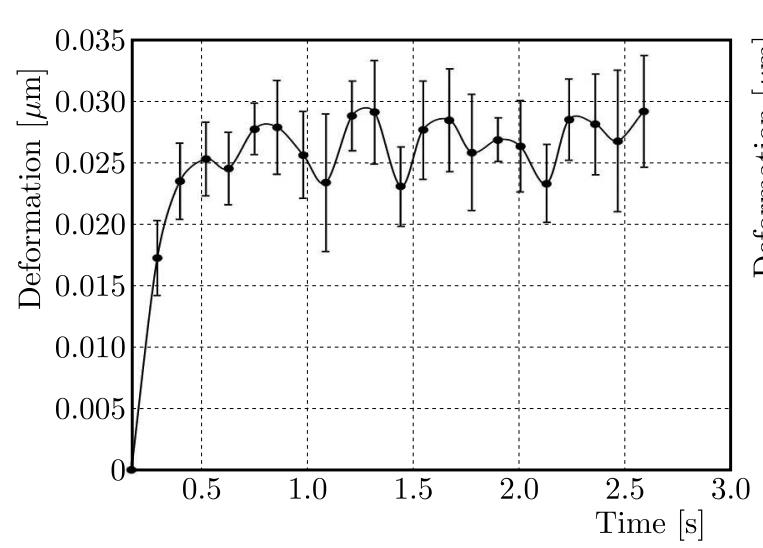

(b)

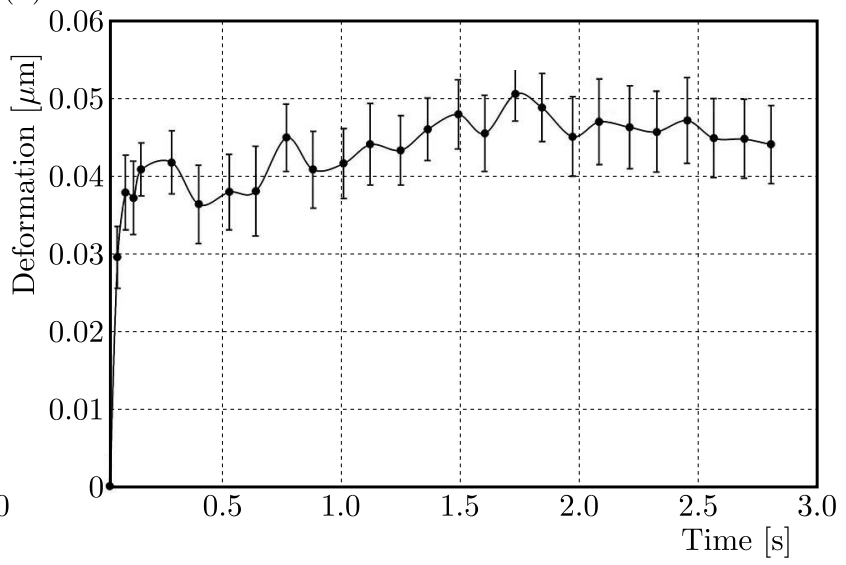

Fig. 2. The average deformation against time from experimental results. Diagram of the Radiation Group with solid behavior (a). Diagram of the Control group with solid behavior (b) 
First, before calculating the static stiffness, the mean displacement-time graphs were used to find the best mechanical model. The graphs were analyzed using Mathematica 9 curve fitting method. Between two mechanical models of Kelvin and the Standard Linear Solid Model, a model with the selected mechanical model for both the radiation and control groups with solid behavior is the standard linear solid model because of the least mean square error.

The stress-strain equation for the standard linear solid model has been specified as

$$
\sigma+\frac{\frac{\eta}{E_{1}}}{1+\frac{E_{2}}{E_{1}}} \dot{\sigma}=\frac{E_{2}}{1+\frac{E_{2}}{E_{1}}} \varepsilon+\frac{\eta}{1+\frac{E_{2}}{E_{1}}} \dot{\varepsilon}
$$

and

$$
\begin{array}{lll}
\varepsilon(t)=\frac{\sigma_{0}}{q_{1}}\left[\frac{1}{\lambda}\left(1-\mathrm{e}^{-\lambda t}\right)+p_{1} \mathrm{e}^{-\lambda t}\right] & \lambda=\frac{q_{0}}{q_{1}} \\
p_{1}=\frac{\frac{\eta}{E_{1}}}{1+\frac{E_{2}}{E_{1}}} \quad q_{0}=\frac{E_{2}}{1+\frac{E_{2}}{E_{1}}} & q_{1}=\frac{\eta}{1+\frac{E_{2}}{E_{1}}}
\end{array}
$$

In these equations, the values $E_{1}, E_{2}$, and $\eta$ represent the coefficients of the springs and the damper, respectively. In order to simplify the main equation, the parameters $\lambda, q_{0}, q_{1}$ and $p_{1}$ have been replaced by the expressions in the strain-strain equation.

After determining the appropriate mechanical model, the curve fitting of the time displacement graph was performed for the standard linear solid model, the results of which are shown in Fig. 3. Also, the results of this curve fitting, which are the coefficients of the spring and the damper, have been specified in Table 1. The correctness of the coefficients obtained from the samples has been determined by the P test (Beaujean et al., 2011).

(a)

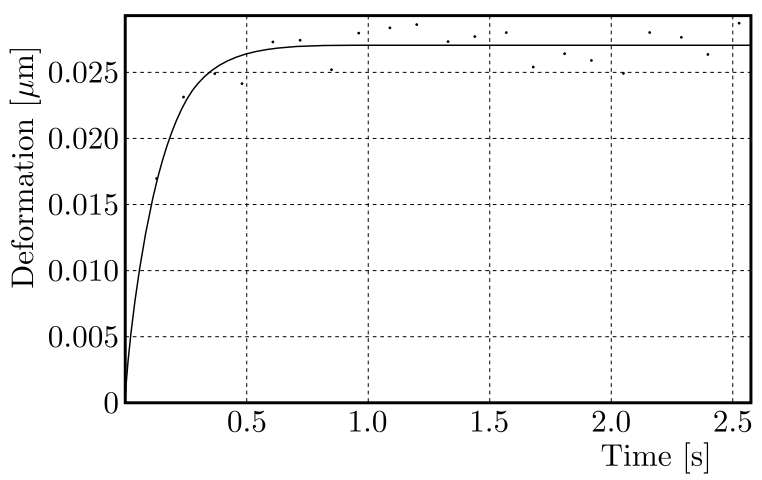

(b)

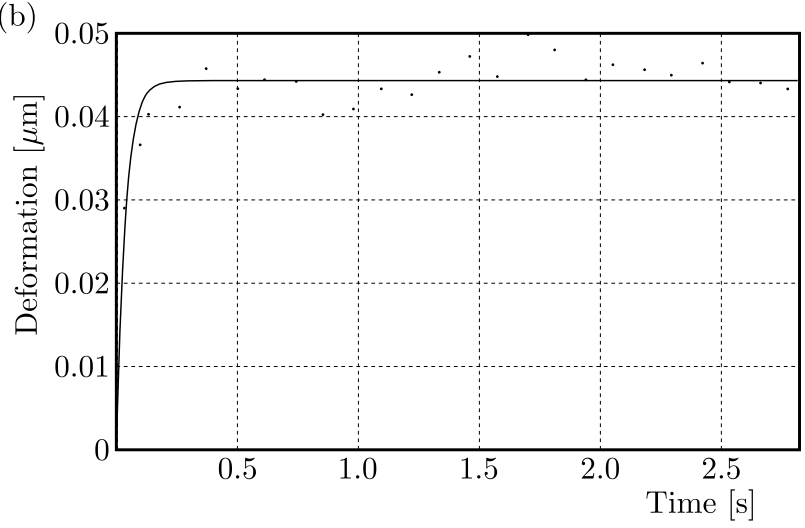

Fig. 3. Differential equation results of the model curve-fitted on experimental data. The radiation group (a). The control group (b)

Table 1. Numerical values of spring and damper coefficients

\begin{tabular}{|c|c|c|c|c|}
\hline Coefficients & \multicolumn{2}{|c|}{ Radiation group } & \multicolumn{2}{c|}{ Control group } \\
\hline \hline$\eta[\mu \mathrm{Nsm}]$ & $1.4889 \cdot 10^{3}$ & $P<0.05$ & $2.6229 \cdot 10^{2}$ & $P<0.05$ \\
\hline$E_{1}[\mu \mathrm{N} / \mathrm{m}]$ & $2.5689 \cdot 10^{6}$ & $P<0.05$ & $3.4742 \cdot 10^{5}$ & $P<0.05$ \\
\hline$E_{2}[\mu \mathrm{N} / \mathrm{m}]$ & $1.1107 \cdot 10^{4}$ & $P<0.05$ & $6.8764 \cdot 10^{3}$ & $P<0.05$ \\
\hline
\end{tabular}

After determining the selected mechanical model and calculating the coefficients of the springs and the damper, static stiffness has been calculated according to Eq. (3.3). In this equation, the value $\sigma_{0}$ is $299 \mathrm{pN}$ (Fig. 4)

$$
D(t)=\frac{\varepsilon(t)}{\sigma_{0}}
$$




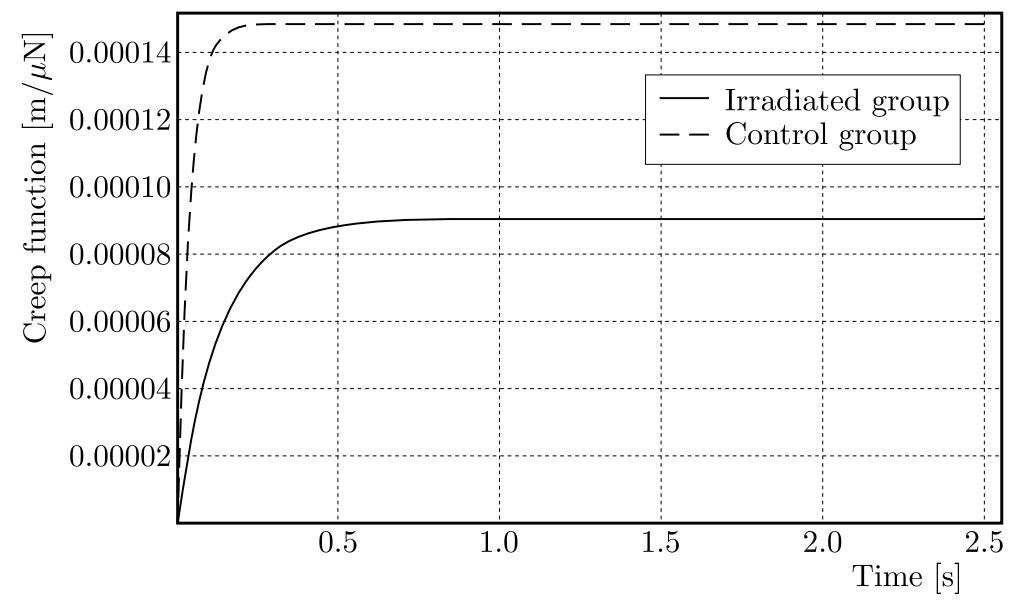

Fig. 4. Diagrams of the creep function of the samples from the radiation group (solid line) and the control group (dotted line)

(a)

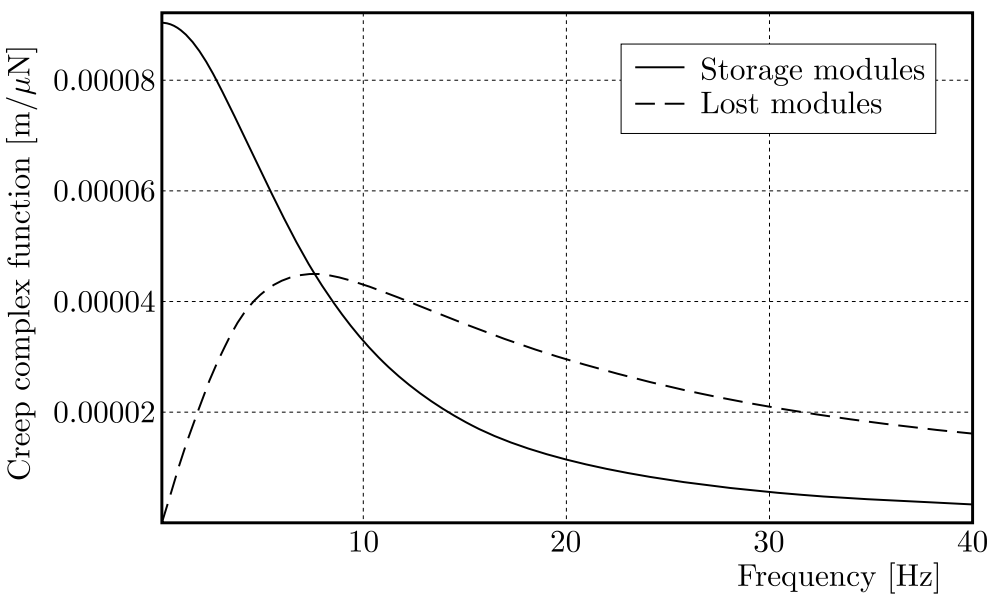

(b)

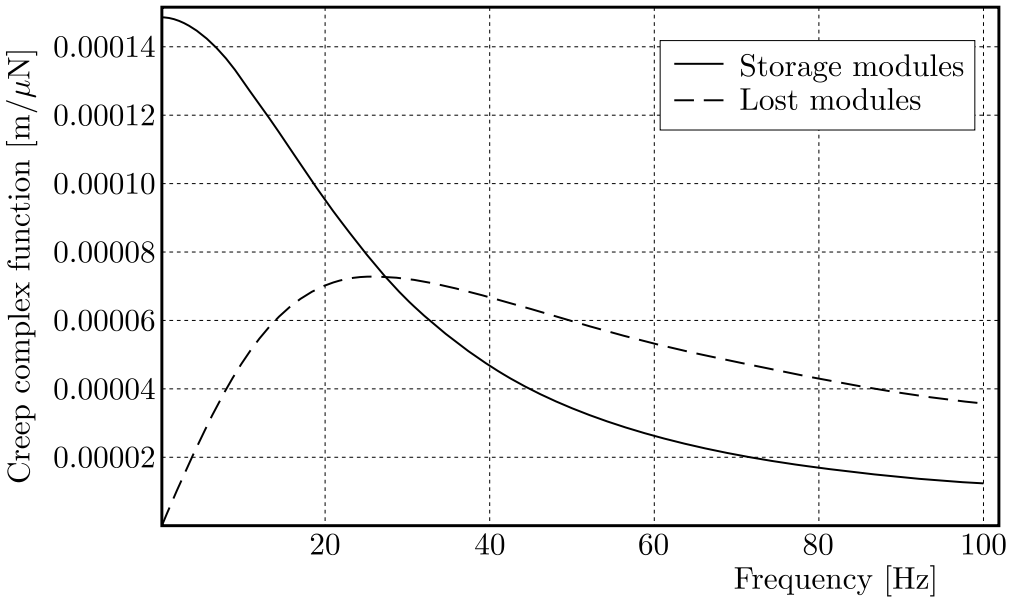

Fig. 5. Diagrams of the complex creep function of the samples. Diagram of the radiation group (a) and the control group (b)

To calculate the dynamic state of the samples, $D *$ (complex creep function) was first drawn for both the radiation and control groups (Fig. 5). The equation related to $D *$ is for materials with solid behavior according to Eqs. (3.4) and (3.5) in which $\omega$ is the loading frequency. This graph, according to Eq. (3.4), has two real and imaginary parts, both of which have been shown separately in the plotted charts 


$$
D^{\omega}=\left(\frac{1}{E_{2}}+\frac{E_{2}}{E_{2}^{2}+\eta_{2}^{2} \omega^{2}}\right)-\mathrm{i}\left(\frac{1}{\eta_{1} \omega}+\frac{\eta_{2} \omega}{E_{2}^{2}+\eta_{2}^{2} \omega^{2}}\right)
$$

and

$$
D^{*}(\omega)=\frac{q_{0}+q_{1} p_{1} \omega^{2}}{q_{0}^{2}+q_{1}^{2} \omega^{2}}-i \frac{\omega\left(q_{0} p_{1}-q_{1}\right)}{q_{0}^{2}+q_{1}^{2} \omega^{2}}
$$

The results of plotting the mean time-displacement graphs (Fig. 2) show that the mean maximum deformation of the membrane in the control group is $0.049 \pm 0.01 \mu \mathrm{m}$. While it was $0.029 \pm 0.01 \mu \mathrm{m}$ for the radiation group. The values indicate a decrease in deformation in the radiation group compared to the control group. The starting point of the creep response of samples is $0.1 \mathrm{~s}$ and $0.2 \mathrm{~s}$ for the control group and the radiation group with the same loading respectively. The results of the fitting curve (Fig. 3) are presented in the table of parameters (Table 1). The comparison of the obtained values indicates the increase of the damping coefficient $\eta$ and the first spring of the model $E_{1}$ after radiation. Also, the coefficient of the second spring $E_{2}$ has decreased. It should be noted that this difference in coefficients is not enough to evaluate the viscoelastic properties. In the next section, for the creep function $D(t)$, which is the static stiffness, the static stiffness of the radiation group was significantly lower than in the control group at all times. For example, in the static loading for $0.5 \mathrm{~s}$, this value for the radiation group is $8.897 \cdot 10^{-5} \mathrm{~m} /(\mu \mathrm{N})$ and for the control group, it is $1.474 \cdot 10^{-4} \mathrm{~m} /(\mu \mathrm{N})$ (Fig. 4). Regarding the frequency analysis of the models, changes in the behavior of the material model are evident concerning the frequency of loading after irradiation. Both groups exhibit solid behavior before the gel point, but they have different gel points. This point for the control group is $27 \mathrm{~Hz}$ and for the radiation group is $7.5 \mathrm{~Hz}$. The behavior of both groups after this frequency is fluid. Also, the complex creep function $D^{*}$, which represents the dynamic stiffness of the model, has reduced in all the post-test frequencies, for example, for a frequency of $1.57 \mathrm{~Hz}$, which is the natural dynamic loading frequency of the jaw and tooth (chewing) (Po et al., 2011). The amount of dynamic stiffness for the control and radiation groups is $1.483 \cdot 10^{-4} \mathrm{~m} /(\mu \mathrm{N})$ and $0.889 \cdot 10^{-4} \mathrm{~m} /(\mu \mathrm{N})$, respectively (Fig. 5).

\section{Discussion}

According to the results of the current test, it can be concluded that the results obtained to determine the effect of X-ray radiation on cell deformation indicate a decrease of this parameter for irradiated cells compared to control cells. This conclusion is important because, according to our assumption and the results of previous studies as well as regarding the ionizing nature of X-ray, it was expected that the structure of the skeletal proteins would change, thereby, the deformation of the sample increased after radiation. This assumption was not seen in our data and, conversely, the deformation of the cell decreased in the radiation group compared to the control group. The main reason for this difference may be due to activation of actin strands in the cytoskeleton. Actin filaments play the central role in cell dynamic behavior according to polymerization. The filaments provide forces for numerous cell actions such as endocytosis, morphogenesis, and migration. Any changes in regulation of actin-binding proteins could affect cellular mechanical activities (Itoh et al., 2005; Saarikangas et al., 2010). This suspicion is consistent with the findings of previous studies. Panzetta et al. (2017) studied the effect of $\mathrm{X}$-rays on mechanical properties of cancerous and healthy cells. They found out that X-rays on both cancerous and healthy cells can activate strands of cytoskeleton and increase mechanical properties of the cytoskeleton (Panzetta et al., 2017).

In addition, the results of our experiment showed a decrease in cellular stiffness in both static and dynamic conditions for irradiated specimens compared to controls. These results are entirely 
consistent with our initial assumption suggesting the ionizing effect on decreasing of the cellular stiffness. In fact, it can be concluded that X-rays have the potential for impact on the structure of macromolecules and cellular structural proteins due to ionizing properties (Sabanero et al., 2016). Therefore, it can be concluded that this is the ionizing effect which has made a change in the structure of the proteins of cytoskeleton and reduced the cell stiffness. It is worth noting that there are many contradictory and consistent results in relation to this conclusion. For example, (Zheng et al., 2015) in their study on the effect of X-rays on the properties of cytoskeleton and biochemical properties of cancer cells of the tongue tissue found that X-rays can reduce Young's modulus of the cells and thus reduce their stiffness from $3.1 \pm 0.1 \mathrm{kPa}$ to $0.9 \pm 0.1 \mathrm{kPa}$ for $100 \mathrm{kVp}$ $\mathrm{X}$-ray. This result is fully consistent with our findings (Zheng et al., 2015).

Also, Zhang et al. (2014) in another study on the effect of ionizing radiations (including $\mathrm{X}$-ray radiation) on the stiffness of erythrocytes cells found that X-rays reduced the amount of Young's modulus of the cells and thus decreased their stiffness from $9.426 \pm 0.66 \mathrm{kPa}$ to $4.986 \pm 0.76 \mathrm{kPa}$. They also measured the morphological differences which indicated that the cell volume declined from $41.036 \pm 7.30 \mu \mathrm{m}^{3}$ to $38.996 \pm 5.95 \mu \mathrm{m}^{3}$ (Zhang et al., 2014). However, a number of previous studies have achieved quite different results. For example, Panzetta et al. (2017) in their experiment on the effect of high energy X-ray radiation on the mechanical properties of cells, showed that this radiation could increase the elasticity modulus and stiffness of the cells from $1.4 \pm 0.04 \mathrm{kPa}$ to $1.8 \pm 0.07 \mathrm{kPa}$ (for 2 Gy after 24 hours) (Panzetta et al., 2017). Also, in their study on the effect of X-ray radiation on the mechanical properties of lymphocyte and platelets, Thomas et al. (2003) reported increasing elasticity modulus and stiffness. In addition to aforementioned, Du et al. (2014) in their study on the effects of X-rays on nerve cells reported an increase in Young's modulus and stiffness of cells after radiation from $0.4 \pm 0.1 \mathrm{kPa}$ to $0.9 \pm 0.1 \mathrm{kPa}$. This difference in our findings and in a number of previous studies may be due to different cell lines or methods of measuring these properties. It should be noted that cells exhibit strain responses against environmental stress over time (Desprat et al., 2005; Selvaggi et al., 2010). It is crucial for the viscoelastic behavior. Therefore, in this paper, the viscoelastic model has been used, and it explains the cellular behavior more properly.

\section{References}

1. Bao G., Suresh S., 2003, Cell and molecular mechanics of biological materials, Nature Materials, 2, 715-725

2. Beaujean F., Caldwell A., Kollár D., Kröninger K., 2011, P-values for model evaluation, Physical Review D, 83, DOI: 10.1103/PhysRevD.83.012004

3. Boeddinghaus R., Whyte A., 2008, Current concepts in maxillofacial imaging, European Journal of Radiology, 66, 396-418, DOI: 10.1016/j.ejrad.2007.11.019

4. Cerqueira E.D.M.M., Meireles J.R.C., Lopes M.A., Junqueira V.C., Gomes-Filho I.S., Trindade S., Machado-Santelli G.M., 2008, Genotoxic effects of X-rays on keratinized mucosa cells during panoramic dental radiography, Dentomaxillofacial Radiology, 37, 398-403. DOI: $10.1259 / \mathrm{dmfr} / 56848097$

5. Desprat N., Richert A., Simeon J., Asnacios A., 2005, Creep function of a single living cell, Biophysical Journal, 88, 2224-2233, DOI: 10.1529/biophysj.104.050278

6. Dobson J., 2008, Remote control of cellular behaviour with magnetic nanoparticles, Nature Nanotechnology, 3, 139-143, DOI: 10.1038/nnano.2008.39

7. Du Y.T., Zhang J., Zheng Q., Li M.X., Liu Y., Zhang B.P., Liu B., Zhang H., Miao G.Y., 2014, Heavy ion and X-ray irradiation alter the cytoskeleton and cytomechanics of cortical neurons, Neural Regeneration Research, 9, 1129-1137, DOI: 10.4103/1673-5374.135315 
8. Health and Physics: A Grade 12 Manitoba Physics Resource for Health and Radiation Physics, 2009, Canadian Cancer Society

9. INGBER D.E., 2002, Mechanical signaling and the cellular response to extracellular matrix in angiogenesis and cardiovascular physiology, Circulation Research, 91, 877-887, DOI: 10.1161/01.RES.0000039537.73816.E5

10. Iтоh T., Erdmann K.S., Roux A., Habermann B., Werner H., De Camilli P., 2005, Dynamin and the actin cytoskeleton cooperatively regulate plasma membrane invagination by BAR and F-BAR proteins, Developmental Cell, DOI: 10.1016/j.devcel.2005.11.005

11. Kalinin A.E., Kajava A.V, Steinert P.M., 2002, Epithelial barrier function: assembly and structural features of the cornified cell envelope, BioEssays, 24,9, 789-800, DOI: 10.1002/bies.10144

12. Kanger J.S., Subramaniam V., van Driel R., 2008, Intracellular manipulation of chromatin using magnetic nanoparticles, Chromosome Research, DOI: 10.1007/s10577-008-1239-1

13. Kollmannsberger P., Fabry B., 2007, High-force magnetic tweezers with force feedback for biological applications, Review of Scientific Instruments, 78, 1-7, DOI: 10.1063/1.2804771

14. Overby D.R., Matthews B.D., Alsberg E., Ingber D.E., 2005, Novel dynamic rheological behavior of individual focal adhesions measured within single cells using electromagnetic pulling cytometry, Acta Biomaterialia, 1, 295-303, DOI: 10.1016/j.actbio.2005.02.003

15. Pan Y., Du X., Zhao F., Xu B., 2012, Magnetic nanoparticles for the manipulation of proteins and cells, Chemical Society Reviews, 41, 2912, DOI: 10.1039/c2cs15315g

16. Panzetta V., Musellav I., Pugliese M., Piccolo C., Pasqua G., Netti P.A., Fusco S., 2017, Effects of high energy X-rays on cell morphology and functions, ENBENG 2017 - 5th Portuguese Meeting on Bioengineering, Proceedings, DOI: 10.1109/ENBENG.2017.7889448

17. Po J.M.C., Kieser J.A., Gallo L.M., Tésenyi A.J., Herbison P., Farella M., 2011, Time-frequency analysis of chewing activity in the natural environment, Journal of Dental Research, 90, 1206-1210, DOI: 10.1177/0022034511416669

18. Preethi N., Chikkanarasaiah N., Bethur S.S., 2016, Genotoxic effects of X-rays in buccal mucosal cells in children subjected to dental radiographs, BDJ Open, 2, 16001, DOI: 10.1038/bdjopen.2016.1

19. Rianna C., Radmacher M., 2016, Cell mechanics as a marker for diseases: Biomedical applications of AFM, AIP Conference Proceedings, 1760, DOI: 10.1063/1.4960276

20. Risi R., Manti L., Perna G., Lasalvia M., Capozzi V., Delfino I., Lepore M., 2012, $\mathrm{X}$-ray radiation-induced effects in human mammary epithelial cells investigated by Raman microspectroscopy, SPIE Photonics Europe, 84272E-84272E-10, DOI: 10.1117/12.921389

21. Rosenbluth M.J., Lam W.A., Fletcher D.A., 2008, Analyzing cell mechanics in hematologic diseases with microfluidic biophysical flow cytometry, Lab on a Chip, 8, 1062, DOI: $10.1039 / \mathrm{b} 802931 \mathrm{~h}$

22. SaArikangas J., Zhao H., Lappalainen P., 2010, Regulation of the actin cytoskeleton-plasma membrane interplay by phosphoinositides, Physiological Reviews, DOI: 10.1152/physrev.00036.2009

23. Sabanero M., Azorín-Vega, J.C., Flores-Villavicencio L.L., Pedro Castruita-Dominguez J., Vallejo M.A., Barbosa-Sabanero G., Cordova-Fraga T., Sosa-Aquino M., 2016, Mammalian cells exposed to ionizing radiation: Structural and biochemical aspects, Applied Radiation and Isotopes, 108, 12-15, DOI: 10.1016/j.apradiso.2015.11.064

24. Selvaggi L., Salemme M., Vaccaro C., Pesce G., Rusciano G., Sasso A., Campanella C., Carotenuto R., 2010, Multiple-Particle-Tracking to investigate viscoelastic properties in living cells, Methods, 51, 1, DOI: 10.1016/j.ymeth.2009.12.008

25. Tanase M., Biais N., Sheetz M., 2007, Magnetic tweezers in cell biology, Methods in Cell Biology, DOI: 10.1016/S0091-679X(07)83020-2 
26. Thomas S., Bolch W., Kao K.J., Bova F., Tran-Son-Tay R., 2003, Effects of X-ray radiation on the rheologic properties of platelets and lymphocytes, Transfusion, 43, 502-508, DOI: 10.1046/j.1537-2995.2003.00360.x

27. VAlEntin J., EDiT., 2007, The 2007 recommendations of the international commission on radiological protection, Annals of the ICRP, 37, 332

28. Verma M., Sonam, Ayub S., 2016, Biological effects of X-rays on X-ray technicians, International Journal of Innovative Research in Science, Engineering and Technology, 18512-18516, DOI: 10.15680/IJIRSET.2016.0510056

29. Zhang B., Liu B., Zhang H., Wang J., 2014, Erythrocyte stiffness during morphological remodeling induced by carbon ion radiation, PLOS One, 9, DOI: 10.1371/journal.pone.0112624

30. Zheng Q., Liu Y., Zhou H.J., Du Y.T., Zhang B.P., Zhang J., Miao G.Y., Liu B., Zhang H., 2015, X-ray radiation promotes the metastatic potential of tongue squamous cell carcinoma cells via modulation of biomechanical and cytoskeletal properties, Human ans Experimental Toxicology, 34, 894-903, DOI: 10.1177/0960327114561664

Manuscript received September 3, 2018; accepted for print May 31, 2019 